\title{
Homology theory for the set-theoretic Yang-Baxter equation and knot invariants from generalizations of quandles
}

\author{
by
J. Scott Carter (Mobile, AL), Mohamed Elhamdadi (Tampa, FL) and Masahico Saito (Tampa, FL)

\begin{abstract}
A homology theory is developed for set-theoretic Yang-Baxter equations, and knot invariants are constructed by generalized colorings by biquandles and YangBaxter cocycles.
\end{abstract}

1. Introduction. The introduction of virtual knots by Kauffman $[24$, $25]$ re-focused attention on algebraic structures that are defined via diagrams. Advantages of using virtual knots were observed for the bracket polynomial [25] and Vassiliev invariants [19]. The fundamental (Wirtinger) groups of virtual knots were studied $[27,34]$ and their geometric interpretations were given [22]. Generalizations of Alexander polynomials were studied in relations to virtual knots $[33,35]$. The theory of racks and quandles (in particular the homology theory thereof) as exposed in $[14,16,17]$ was used to define state-sum invariants for knotted surfaces, as well as for classical and virtual knots [5]. A generalization of quandles, called biquandles, is proposed in [26]. Examples include a generalized Burau matrix used in [33] and [35]. The set-theoretic solutions to the Yang-Baxter equations are studied in detail in the papers $[11,12,29,36]$. Their affine solutions appear among our birack matrices. Some of these solutions also appeared in [35] and [10].

In this paper a homology theory for the YBE is constructed, and cocycles are used to define knot invariants via colorings of (virtual) knot diagrams by biquandles and a state-sum formulation.

This paper is organized into two sections; Section 2 develops algebraic theories, and Section 3 gives applications to knot theory. In Section 2, the Yang-Baxter sets are reviewed and colorings of cubical complex by Yang-

2000 Mathematics Subject Classification: 57M25, 55N35.

Research of J. S. Carter supported in part by NSF Grant DMS \#0301095.

Research of M. Saito supported in part by NSF Grant DMS \#0301089. 
Baxter sets are considered in Section 2.1, for developing homology theories for the Yang-Baxter sets in Section 2.2. Obstruction and extension cocycles are investigated in Section 2.3 along the lines of group and quandle cohomology theories [5]. In Section 3, after a review on biquandles and their colorings in Section 3.1, the cocycle invariants are defined using the YangBaxter cohomology theory, and computations and applications are given in Section 3.3.

\section{Homology theory for set-theoretic Yang-Baxter equations}

2.1. Yang-Baxter sets and their colorings. Let $R=\left(R_{1}, R_{2}\right)$ be a solution to the set-theoretic Yang-Baxter equation on a set $X$, that is, an invertible mapping $R: X \times X \rightarrow X \times X$, written componentwise as $R\left(x_{1}, x_{2}\right)=$ $\left(R_{1}\left(x_{1}, x_{2}\right), R_{2}\left(x_{1}, x_{2}\right)\right)$ for $x_{1}, x_{2} \in X$, satisfies the relation

$$
(R \times 1)(1 \times R)(R \times 1)=(1 \times R)(R \times 1)(1 \times R),
$$

where 1 denotes the identity map. The set $X$ with a solution $R$ to the Yang-Baxter equation, $(X, R)$, is called a Yang-Baxter set. Set-theoretic Yang-Baxter equations have been studied in [11, 12, 29, 36], for example.

EXAMPLE 2.1. Let $k$ be a commutative ring with 1 and with units $s$ and $t$ such that $(1-s)(1-t)=0$. Then $R=\left[\begin{array}{cc}1-s & s \\ t & 1-t\end{array}\right]$ satisfies the YBE. In particular, for any commutative ring $F$ with 1 , let

$$
k=F\left[s^{ \pm 1}, t^{ \pm 1}\right] /(1-s)(1-t) .
$$

Then the above defined $R$ satisfies the conditions. More specifically, $\mathbb{Z}_{q}$ becomes a YB set for integers $s$ and $t$, where $q=|(1-s)(1-t)|$ if $s$ and $t$ are units in $\mathbb{Z}_{q}$.

Another similar example is constructed from matrices. Let $R: X \times X \rightarrow$ $X \times X$ be defined by a matrix $R=\left[\begin{array}{cc}E-Y & Y \\ Z & E-Z\end{array}\right]$ with invertible matrices $Y$ and $Z$ such that $Y Z=Z Y$ and $(E-Y)(E-Z)=0$. Then $R$ satisfies the YBE. Specifically, for any commutative ring $k$ with $1, Y=\left[\begin{array}{ll}1 & s \\ 0 & 1\end{array}\right]$ and $Z=\left[\begin{array}{ll}1 & t \\ 0 & 1\end{array}\right]$ for any $s, t \in k$ give a solution to the YBE. In [3] further examples of solutions to the YBE are given.

Let $\mathcal{I}_{n}$ be the $n$-dimensional cube $I^{n}$ regarded as a $\mathrm{CW}$ (cubical) complex, where $I=[0,1]$ and $n$ is a positive integer. Denote the $k$-skeleton of $\mathcal{I}_{n}$ by $\mathcal{I}_{n}^{(k)}$. Every $k$-dimensional face of $\mathcal{I}_{n}$, for any positive integer $k$, is another $k$-dimensional cube. Give the orientation for each $k$-face to be the one defined from the order of the coordinate axes. Every $k$-face $\sigma$ is regarded as having this orientation fixed, and the same $k$-face with the opposite orientation is denoted by $-\sigma$. In particular, note that every 2 -face can be written as

$$
\left\{\varepsilon_{1}\right\} \times \cdots \times\left\{\varepsilon_{i-1}\right\} \times I_{i} \times\left\{\varepsilon_{i+1}\right\} \times \cdots \times\left\{\varepsilon_{j-1}\right\} \times I_{j} \times\left\{\varepsilon_{j+1}\right\} \times \cdots \times\left\{\varepsilon_{n}\right\}
$$


for some $i$ and $j, 1 \leq i<j \leq n$, where $\varepsilon_{k}=0$ or 1 , and $I_{i}, I_{j}$ denote the $i$ th, $j$ th factors of a copy of $I$, respectively. We abbreviate parentheses for simplicity. Similarly, when the number 0 or 1 is placed at the $i$ th factor, we denote it by $0_{i}$ or $1_{i}$, respectively.

Definition 2.2. The (Yang-Baxter) coloring of $\mathcal{I}_{n}$ by a Yang-Baxter set $(X, R)$ is a map $L: E\left(\mathcal{I}_{n}\right) \rightarrow X$, where $E\left(\mathcal{I}_{n}\right)$ denotes the set of edges (1-faces) of $\mathcal{I}_{n}$, with each edge oriented as above, such that if

$$
\begin{aligned}
& L\left(\varepsilon_{1} \times \cdots \times I_{i} \times \cdots \times 0_{j} \times \cdots \times \varepsilon_{n}\right)=x, \\
& L\left(\varepsilon_{1} \times \cdots \times 1_{i} \times \cdots \times I_{j} \times \cdots \times \varepsilon_{n}\right)=y,
\end{aligned}
$$

then

$$
\begin{aligned}
& L\left(\varepsilon_{1} \times \cdots \times 0_{i} \times \cdots \times I_{j} \times \cdots \times \varepsilon_{n}\right)=R_{1}(x, y), \\
& L\left(\varepsilon_{1} \times \cdots \times I_{i} \times \cdots \times 1_{j} \times \cdots \times \varepsilon_{n}\right)=R_{2}(x, y) .
\end{aligned}
$$

To see how many Yang-Baxter colorings $\mathcal{I}_{n}$ admits, we specify the initial path in $\mathcal{I}_{n}$, i.e., the sequence of edges of $\mathcal{I}_{n},\left(e_{1}, \ldots, e_{n}\right)$, where

$$
\begin{aligned}
& e_{1}=I_{1} \times 0_{2} \times \cdots \times 0_{n}, \\
& e_{2}=1_{1} \times I_{2} \times 0_{3} \times \cdots \times 0_{n}, \\
& \quad \vdots \\
& e_{n}=1_{1} \times \cdots \times 1_{n-1} \times I_{n} .
\end{aligned}
$$

Note that the orientations of the edges $e_{i}$ of the initial path are consistent in the sense that the terminal point of $e_{i}$ is the initial point of $e_{i+1}$, for all $i=1, \ldots, n-1$.

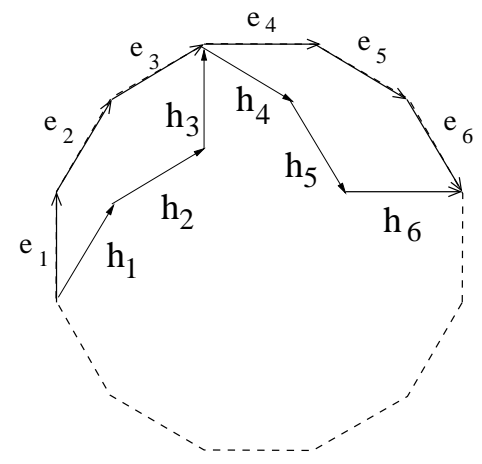

Fig. 1. The initial path of $\mathcal{I}_{6}$

For a subcube

$$
C=\varepsilon_{1} \times \cdots \times I_{j_{1}} \times \cdots \times I_{j_{2}} \times \cdots \times I_{j_{k}} \times \cdots \times \varepsilon_{n}
$$

of dimension $k$, identify $C$ with $\mathcal{I}_{k}$ by the obvious map sending the $h$ th factor of $I$ in $I^{k}$ to $I_{j_{h}}$ in $C$. The orientations are preserved by this identification. Then the sequence of edges of $C$ corresponding to the initial path of $\mathcal{I}_{k}$ under 
this identification is called the initial path of $C$. In Fig. 1, a projection of $\mathcal{I}_{6}$ is depicted, and its initial path is labeled by $e_{1}, \ldots, e_{6}$ along the top edges.

Lemma 2.3. Let $(X, R)$ be a Yang-Baxter set, and $\left(e_{1}, \ldots, e_{n}\right)$ be the initial path of $\mathcal{I}_{n}$. For any $n$-tuple of elements of $X,\left(x_{1}, \ldots, x_{n}\right)$, there exists a unique Yang-Baxter coloring $L$ of $\mathcal{I}_{n}$ by $(X, R)$ such that $L\left(e_{i}\right)=x_{i}$ for all $i=1, \ldots, n$.

Proof. A sequence $\left(h_{1}, \ldots, h_{n}\right)$ of edges in $\mathcal{I}_{n}$ is called a complete path in $\mathcal{I}_{n}$ if the initial point (with respect to the given orientation of each edge) of $h_{1}$ is $0_{1} \times \cdots \times 0_{n}$, the terminal point of $h_{n}$ is $1_{1} \times \cdots \times 1_{n}$, and the initial point of $h_{i+1}$ matches the terminal point of $h_{i}$ for all $1<i<n-1$. For example, the initial path $\left(e_{1}, \ldots, e_{n}\right)$ is a complete path, as is the sequence of edges

$$
\begin{aligned}
h_{i} & =e_{i} \quad \text { for } i \neq j, j+1, \\
h_{j} & =(\underbrace{1, \ldots, 1}_{j-1}, 0, I_{j+1}, \underbrace{0, \ldots, 0}_{n-j-1}), \\
h_{j+1} & =(\underbrace{1, \ldots, 1}_{j-2}, 0, I_{j}, \underbrace{0, \ldots, 0}_{n-j}) .
\end{aligned}
$$

In Fig. 1, (the projection of) another example of a complete path $\left(h_{1}, \ldots, h_{6}\right)$ is depicted.

The complete path $\left(h_{1}, \ldots, h_{n}\right)$ defined above is obtained from $\left(e_{1}, \ldots, e_{n}\right)$ by sweeping the square

$$
(\underbrace{1, \ldots, 1}_{j-1}, I_{j} \times I_{j+1}, \underbrace{0, \ldots, 0}_{n-j}) .
$$

We describe sweeping a square in full generality as follows. Suppose that

$$
\begin{aligned}
h_{j} & =\varepsilon_{1} \times \cdots \times I_{\alpha} \times \cdots \times 0_{\beta} \times \cdots \times \varepsilon_{n}, \\
h_{j+1} & =\varepsilon_{1} \times \cdots \times 1_{\alpha} \times \cdots \times I_{\beta} \times \cdots \times \varepsilon_{n}, \\
h_{j}^{\prime} & =\varepsilon_{1} \times \cdots \times 0_{\alpha} \times \cdots \times I_{\beta} \times \cdots \times \varepsilon_{n}, \\
h_{j+1}^{\prime} & =\varepsilon_{1} \times \cdots \times I_{\alpha} \times \cdots \times 1_{\beta} \times \cdots \times \varepsilon_{n},
\end{aligned}
$$

and $h_{i}=h_{i}^{\prime}$ for $i \neq j, j+1$. Then the complete path $\left(h_{1}^{\prime}, \ldots, h_{n}^{\prime}\right)$ is said to be obtained from the complete path $\left(h_{1}, \ldots, h_{n}\right)$ by sweeping a square. Note that this is not a symmetric relation. This situation is also denoted by $\left(h_{1}, \ldots, h_{n}\right) \Rightarrow\left(h_{1}^{\prime}, \ldots, h_{n}^{\prime}\right)$. The two paths $\left(h_{1}, \ldots, h_{n}\right)$ and $\left(h_{1}^{\prime}, \ldots, h_{n}^{\prime}\right)$ bound the square

$$
\left(\varepsilon_{1} \times \cdots \times I_{\alpha} \times \cdots \times I_{\beta} \times \cdots \times \varepsilon_{n}\right) .
$$

This square is called the square swept by the paths. A square swept by paths looks like the square in Fig. 2, where $\left(h_{1}, \ldots, h_{n}\right)$ goes through the top two edges and $\left(h_{1}^{\prime}, \ldots, h_{n}^{\prime}\right)$ goes through the bottom two edges. 
If there are paths $\mathcal{P}_{k}=\left(h_{1}^{(k)}, \ldots, h_{n}^{(k)}\right)$ for $k=0, \ldots, m$ such that $\mathcal{P}=$ $\mathcal{P}_{0} \Rightarrow \mathcal{P}_{1} \Rightarrow \cdots \Rightarrow \mathcal{P}_{m}=\mathcal{P}^{\prime}$, then $\mathcal{P}^{\prime}$ is said to be obtained from $\mathcal{P}$ by sweeping squares and we write $\mathcal{P} \Longrightarrow \mathcal{P}^{\prime}$ (the double arrow in notation is longer in this case). By the definition of a Yang-Baxter coloring, if the values $L\left(h_{i}\right)$ are specified for all $i=1, \ldots, n$, and $\left(h_{i}\right) \Longrightarrow\left(h_{j}^{\prime}\right)$, then there exist values $L\left(h_{j}^{\prime}\right)$ such that the Yang-Baxter coloring condition is satisfied for each square that is swept.

We now give a bijective correspondence between the set of complete paths and the symmetric group $\Sigma_{n}$. For a given complete path $\mathcal{P}=$ $\left(h_{1}, \ldots, h_{n}\right)$, define a permutation $\sigma=\sigma(\mathcal{P}) \in \Sigma_{n}$ by

$$
h_{j}=\varepsilon_{1} \times \cdots \times I_{\sigma(j)} \times \cdots \times \varepsilon_{n} .
$$

That is, $\sigma$ is defined so that the $j$ th segment of $\mathcal{P}$ is parallel to the $\sigma(j)$ th coordinate axis.

Conversely, given a permutation $\sigma \in \Sigma_{n}$, we construct a unique complete path $\mathcal{P}=\mathcal{P}(\sigma)=\left(h_{1}(\sigma), \ldots, h_{n}(\sigma)\right)$ such that $\sigma(\mathcal{P})=\sigma$ as follows:

$$
\begin{aligned}
h_{1}(\sigma) & =\left(0, \ldots, I_{\sigma(1)}, \ldots, 0\right), \\
h_{2}(\sigma) & =(0, \ldots, \underbrace{1}_{\sigma(1)}, \ldots, I_{\sigma(2)}, \ldots, 0), \\
& \vdots \\
h_{k}(\sigma) & =\left(\varepsilon_{1}(\sigma(k)), \ldots, I_{\sigma(k)}, \ldots, \varepsilon_{n}(\sigma(k))\right) .
\end{aligned}
$$

The values of $\varepsilon_{j}(\sigma(k))$ are either 0 or 1 . The value 1 is achieved in the $\sigma(1)$ through $\sigma(k-1)$ coordinates. The remaining values are 0 . This completes the construction of a bijection.

Now we show that any complete path $\mathcal{P}$ can be obtained from the initial path $\mathcal{P}_{0}=\left(e_{1}, \ldots, e_{n}\right)$ by sweeping squares, i.e. $\mathcal{P}_{0} \Longrightarrow \mathcal{P}$. We prove this inductively by considering the permutations $\sigma(\mathcal{P})$ associated to the paths. First we introduce some notation on permutations. Write the permutation $\left(\begin{array}{cccc}1 & 2 & \ldots & n-1 \\ i_{1} & i_{2} & \ldots & i_{n-1}\end{array}\right) \in \Sigma_{n-1}$ as $\left(i_{1}, i_{2}, \ldots, i_{n-1}\right)$. For two permutations $\sigma_{1}, \sigma_{2} \in \Sigma_{n}$, write $\sigma_{1} \Rightarrow \sigma_{2}$ if there is a transposition $(i, j)$ such that $\sigma_{2}=(i, j) \sigma_{1}$. For example, elements of $\Sigma_{3}$ have the following relations:

$$
\begin{array}{ccccc}
(123) & \Rightarrow & (132) & \Rightarrow & (312) \\
\Downarrow & & \Downarrow & & \Downarrow \\
(213) & \Rightarrow & (231) & \Rightarrow & (321)
\end{array}
$$

By definition, if $\mathcal{P}_{1} \Rightarrow \mathcal{P}_{2}$ for two complete paths, then $\sigma\left(\mathcal{P}_{1}\right) \Rightarrow \sigma\left(\mathcal{P}_{2}\right)$. Conversely, if $\sigma_{1} \Rightarrow \sigma_{2}$, then $\mathcal{P}_{1}\left(\sigma_{1}\right) \Rightarrow \mathcal{P}_{2}\left(\sigma_{2}\right)$. Indeed, $\mathcal{P}_{2}\left(\sigma_{2}\right)$ is obtained from $\mathcal{P}_{1}\left(\sigma_{1}\right)$ by sweeping the square of the form $\left(\varepsilon_{1}, \ldots, I_{i}, \ldots, I_{j}, \ldots, \varepsilon_{n}\right)$, where $\sigma_{2}=(i, j) \sigma_{1}$. Note that every permutation can be obtained from the identity by a sequence of such transpositions. It follows that any complete path $\mathcal{P}=\left(h_{1}, \ldots, h_{n}\right)$ is obtained from the initial path $\left(e_{1}, \ldots, e_{n}\right)$ by sweeping squares: $\left(e_{1}, \ldots, e_{n}\right) \Longrightarrow\left(h_{1}, \ldots, h_{n}\right)$. 
Also note that each edge in the $n$-cube is an edge in some complete path. For a given edge $\left(\varepsilon_{1}, \ldots, I_{j}, \ldots, \varepsilon_{n}\right)$ consider those values of $k$ for which $\varepsilon_{k}=1$. Then connect the given edge back to $(0,0, \ldots, 0)$ by a sequence of edges parallel to $I_{k}$. Connect the given edge forward to $(1,1, \ldots, 1)$ by intervals in the remaining coordinates. Since every edge is an edge on some complete path, and since every complete path can be obtained from the initial path by sweeping squares, we have a Yang-Baxter coloring on each edge.

To prove uniqueness, we use projections of knot diagrams, and we need the following set-up. Consider the path $\left(f_{1}, \ldots, f_{n}\right)$, where

$$
\begin{aligned}
f_{1} & =0_{1} \times \cdots \times 0_{n-1} \times I_{n}, \\
f_{2} & =0_{1} \times \cdots \times I_{n-1} \times 1_{n}, \\
& \vdots \\
f_{n} & =I_{1} \times 1_{2} \times \cdots \times 1_{n},
\end{aligned}
$$

which we call the terminal path of $\mathcal{I}_{n}$. In Fig. 1, the bottom edges form the terminal path, and the union of the initial and terminal paths forms the boundary of the $n$-gon, which is a projection of $\mathcal{I}_{n}$ into the plane. The union of squares swept by a sequence $\left(e_{i}\right) \Longrightarrow\left(f_{i}\right)$ from the initial path to the terminal path is a union of parallelograms tiling the $2 n$-gon (see Fig. 4 for such tilings for $n=4)$. The union of squares swept by any path $\left(e_{i}\right) \Longrightarrow\left(h_{i}\right)$ for any given path $\left(h_{i}\right)$ is a subset of such a tiling of the whole $2 n$-gon.

Recall the knot diagrams dual to parallelograms as depicted in Fig. 2. Consider the projections of such knot diagrams. The union of squares swept by a sequence $\left(e_{i}\right) \Longrightarrow\left(f_{i}\right)$ gives rise to a projection of dual knot diagrams that consist of generic straight lines on the plane. Hence there is a one-toone correspondence between the set of tilings of an $2 n$-gon in the plane by parallelograms, and the set of generic straight $n$ lines on the plane. In this correspondence, the Yang-Baxter relation corresponds to a Reidemeister type III move, as depicted in Fig. 3. This is a rearrangement of tilings of 3 adjacent parallelograms. We call this a Yang-Baxter rearrangement.

It remains to be seen that for the tilings $T_{1}$ and $T_{2}$ corresponding to given two sequences $S_{1}:\left(e_{i}\right) \Longrightarrow\left(f_{i}\right)$ and $S_{2}:\left(e_{i}\right) \Longrightarrow\left(f_{i}\right)$ respectively, there is a sequence of Yang-Baxter rearrangements $S_{1} \stackrel{a_{1}}{\rightarrow} \ldots \stackrel{a_{k}}{\rightarrow} S_{2}$.

Let $\left(\ell_{1}, \ldots, \ell_{n}\right)$ be the set of straight lines on the plane $\mathbb{R}^{2} \times\{0\}$ corresponding to $T_{1}$, and let $\left(\ell_{1}^{\prime}, \ldots, \ell_{n}^{\prime}\right)$ be those in $\mathbb{R}^{2} \times\{1\}$ corresponding to $T_{2}$. Consider the planes $\left(P_{1}, \ldots, P_{n}\right)$ in $\mathbb{R}^{2} \times[0,1] \subset \mathbb{R}^{3}$ such that each plane $P_{i}(i=1, \ldots, n)$ is spanned by $\ell_{i}$ and $\ell_{i}^{\prime}$. By a small homotopy if necessary, it is assumed that $\left(P_{1}, \ldots, P_{n}\right)$ do not have intersections among more than three planes, and that the triple points are located at distinct levels with respect to the height (the second factor of $\mathbb{R}^{2} \times[0,1]$ ) direction. Such a triple 
point corresponds to a Reidemeister type III move, and to a Yang-Baxter rearrangement in the dual tilings.

Let $t_{0}=0<t_{1}<\cdots<t_{v}=1$ be numbers such that there is exactly one triple point between $\mathbb{R}^{2} \times\left\{t_{j}\right\}$ and $\mathbb{R}^{2} \times\left\{t_{j+1}\right\}$. Then the dual tilings of $P_{i} \cap \mathbb{R}^{2} \times\left\{t_{i}\right\}$ give a sequence of Yang-Baxter rearrangements from $\left(\ell_{i}\right)$ to $\left(\ell_{i}^{\prime}\right)$, as desired.

2.2. Yang-Baxter homology theory. Let $(X, R)$ be a Yang-Baxter set. Let $C_{n}^{\mathrm{YB}}(X)$ be the free abelian group generated by $n$-tuples $\left(x_{1}, \ldots, x_{n}\right)$ of elements of $X$.

Consider a Yang-Baxter coloring $L$ of $\mathcal{I}_{n}$ with $L\left(e_{i}\right)=x_{i}$ for all $i=$ $1, \ldots, n$, which exists uniquely by Lemma 2.3. This $L$ is fixed until the end of the definition of the chain complex. Consider any $k$-face (subcube) $\mathcal{J}$ of $\mathcal{I}_{n}$. Let $\left(f_{1}, \ldots, f_{k}\right)$ be the initial path of $\mathcal{J}$. Then there is a unique $k$-tuple of elements of $X,\left(y_{1}, \ldots, y_{k}\right)$, such that $L\left(f_{j}\right)=y_{j}$ for all $j=1, \ldots, k$. Denote this situation by $L(\mathcal{J})=\left(y_{1}, \ldots, y_{k}\right)$.

Let $\partial_{n}^{C}$ denote the $n$-dimensional boundary map in cubical homology theory. Thus $\partial_{n}^{C}\left(\mathcal{I}_{n}\right)=\sum_{i=1}^{2 n} \varepsilon_{i} \mathcal{J}_{i}$, where $\mathcal{J}_{i}$ is an $(n-1)$-face and $\varepsilon_{i}= \pm 1$ depending on whether the orientation of $\mathcal{J}_{i}$ matches the induced orientation on $\mathcal{J}_{i}$ or not. For the induced orientation, we take the convention that the inward pointing normal to an $(n-1)$-face appears last in a sequence of vectors that specifies an orientation, and the orientation of the $(n-1)$-face is chosen so that this sequence agrees with the orientation of the $n$-cube. In particular, $C=I_{1} \times \cdots \times I_{n-1} \times\{0\}$ has a compatible orientation.

Define a homomorphism $\partial_{n}: C_{n}^{\mathrm{YB}}(X) \rightarrow C_{n-1}^{\mathrm{YB}}(X)$ by $\partial_{n}\left(\left(x_{1}, \ldots, x_{n}\right)\right)=$ $\sum_{i=1}^{2 n} \varepsilon_{i} L\left(C_{i}\right)$. Since $\partial_{n-1}^{C} \circ \partial_{n}^{C}=0$, we have $\partial_{n-1} \circ \partial_{n}=0$, and $\left\{\partial_{n}\right\}$ defines a chain complex $\left(C_{*}^{\mathrm{YB}}(X), \partial_{n}\right)$. Define as usual the homology groups, homology groups with an abelian coefficient group $A$, cochain groups, cohomology groups with an abelian coefficient group $A$, and denote them by $H_{*}^{\mathrm{YB}}(X), H_{*}^{\mathrm{YB}}(X ; A), C_{\mathrm{YB}}^{*}(X ; A), H_{\mathrm{YB}}^{*}(X ; A)$, respectively. We also call cycles and cocycles in this homology theory Yang-Baxter cycles and cocycles, respectively.

Definition 2.4. The homology and cohomology theories defined above are called the homology and cohomology theories of the set-theoretic YangBaxter equation.

We exhibit explicit formulas of the boundary homomorphisms for low dimensions.

Example 2.5. Let $(X, R)$ be a Yang-Baxter set. In Fig. 2, the 2-dimensional cube $\mathcal{I}_{2}$ is depicted. The top two edges form the initial path, and the edges are colored by $x, y \in X$. The bottom edges are colored by $R_{1}(x, y)$ and $R_{2}(x, y)$, so that this assignment indeed defines a Yang-Baxter coloring 


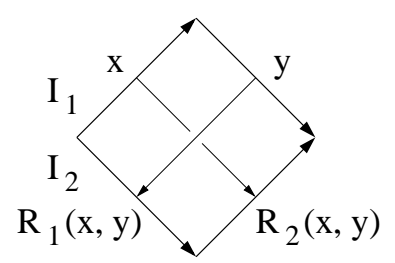

Fig. 2. 2-dimensional boundary homomorphism

of a square. Thus the boundary homomorphism in this case is given by

$$
\partial_{2}(x, y)=(x)+(y)-\left(R_{1}(x, y)\right)-\left(R_{2}(x, y)\right) .
$$

In this square, a correspondence between a square and a positive crossing point used for classical knot diagrams is depicted. We use this correspondence in the following examples.

EXAMPLE 2.6. In Fig. 3, the 3-dimensional cube $\mathcal{I}_{3}$ is depicted at the top of the figure. The top three paths form the initial path, and the elements $x, y, z$ are assigned. The cube, as depicted in the figure, consists of three front faces and three back faces from the reader's perspective. In the top figure of the cube, the three back faces are located behind the three front faces, and thus depicted by dotted lines. In the bottom of the figure, the front faces (left) and the back faces (right) are depicted separately.

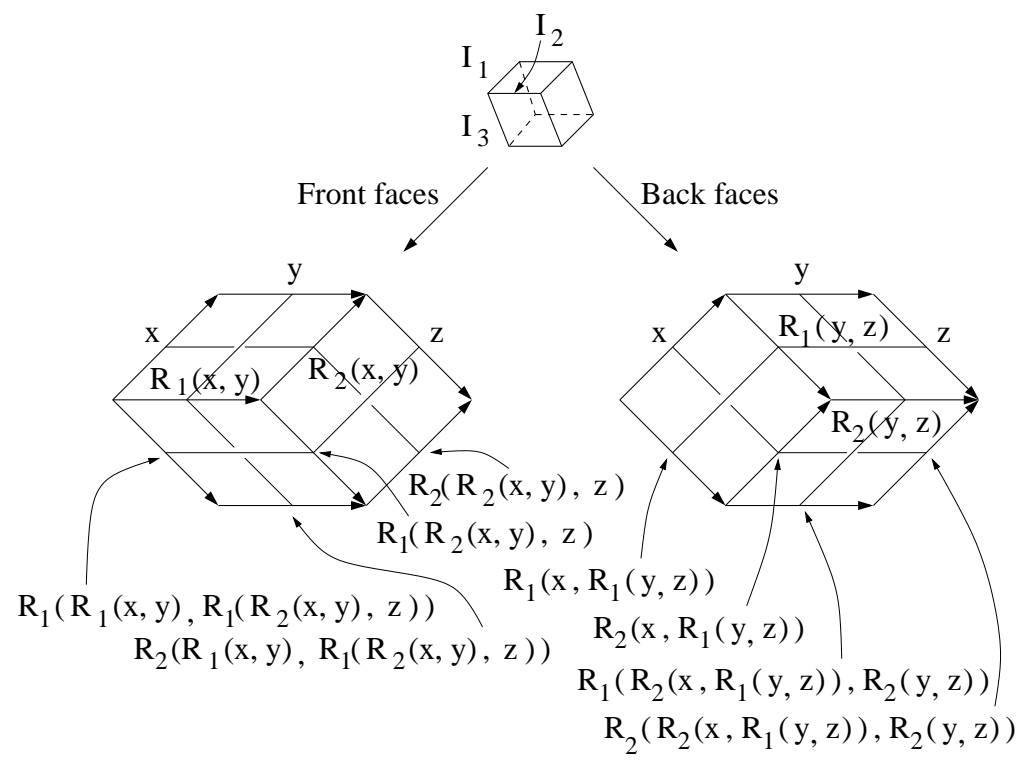

Fig. 3. 3-dimensional boundary homomorphism

The front faces determine, via the condition of a Yang-Baxter coloring, the colors assigned to all the edges of the front faces as depicted. The same 
is true for the edges of the back faces. There are six edges that are common for both front and back faces: three edges of the initial path (labeled by $x, y, z)$ at the top, and three edges at the bottom. The expressions of the colors assigned to these bottom three edges obtained from front faces and those obtained from back faces are different, as depicted in the figure. These are, of course, the same elements in $X$ for each edge, since $R$ is a YangBaxter solution, and the equalities that these elements are the same indeed are equivalent to the condition that $R$ is a solution. The relation between cubes and crossings is again depicted in the bottom figure, and it is seen that the Yang-Baxter equation corresponds to the type III Reidemeister move, as known in knot theory. The boundary homomorphism in this case is given by

$$
\begin{aligned}
\partial_{3}(x, y, z)= & (x, y)+\left(R_{2}(x, y), z\right)+\left(R_{1}(x, y), R_{1}\left(R_{2}(x, y), z\right)\right) \\
& -\left\{(y, z)+\left(x, R_{1}(y, z)\right)+\left(R_{2}\left(x, R_{1}(y, z)\right), R_{2}(y, z)\right)\right\} .
\end{aligned}
$$

In terms of knot diagrams, it is seen that each colored crossing contributes a 2 -chain (pair) $(\alpha, \beta)$, and the Reidemeister type III move (before - after) gives the boundary map.

Example 2.7. In Fig. 4, the 4-dimensional case is depicted. A triple $\left(x_{1}, x_{2}, x_{3}\right) \in C_{3}^{\mathrm{YB}}(X)$ is represented by a cube depicted in Fig. 3 whose initial path $\left(e_{1}, e_{2}, e_{3}\right)$ is colored by $\left(x_{1}, x_{2}, x_{3}\right)$. As in Fig. 3, such a cube is depicted by the three front faces (left) and three back faces (right). In Fig. 4 left and right sides, the four front and back 3-faces of $\mathcal{I}_{4}$ are depicted respectively, by exhibiting each 3 -face (cube) by showing the front three and back three.

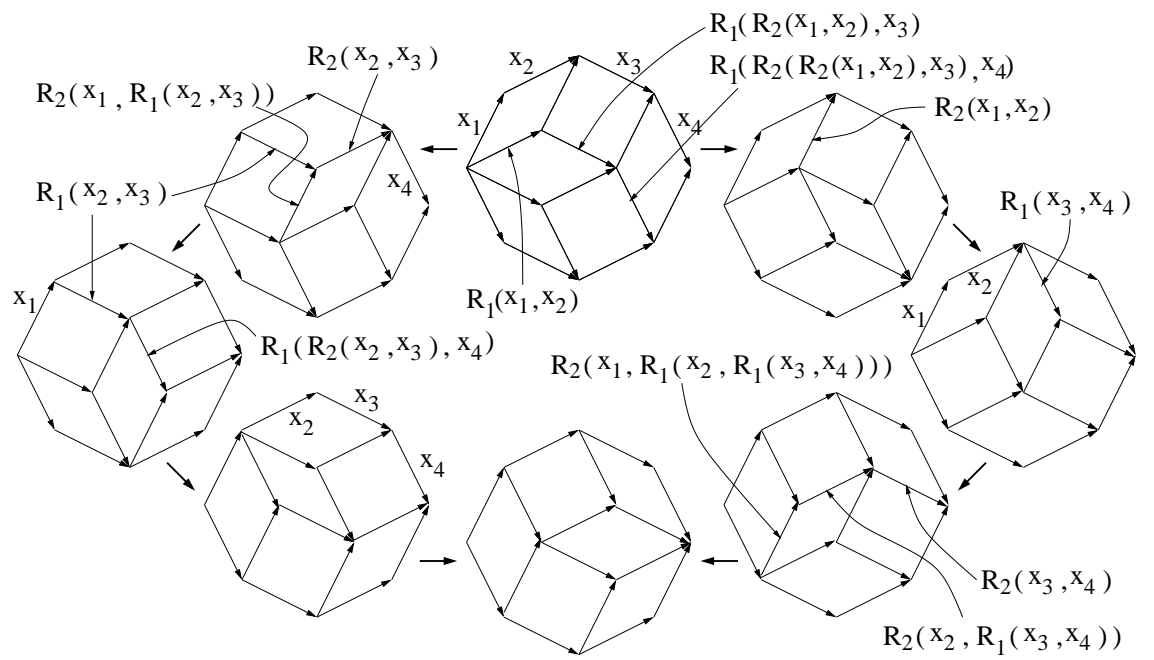

Fig. 4. 4-dimensional boundary homomorphism 
For example, the change (left to right) of Fig. 3 happens for a cube with initial path colored by $\left(x_{1}, x_{2}, x_{3}\right)$ as the first step in the left of Fig. 4 (from top right to top middle), contributing the term $\left(x_{1}, x_{2}, x_{3}\right)$ in $\partial_{4}\left(x_{1}, x_{2}, x_{3}, x_{4}\right)$. On the other hand, in the right of Fig. 4 , the first change is from top left to top middle, which is applied to the cube with initial path colored by $\left(R_{1}\left(x_{1}, x_{2}\right), R_{1}\left(R_{2}\left(x_{1}, x_{2}\right), x_{3}\right), R_{1}\left(R_{2}\left(R_{2}\left(x_{1}, x_{2}\right), x_{3}\right), x_{4}\right)\right)$, the first negative term. From the figure we obtain

$$
\begin{aligned}
\partial_{4}\left(x_{1},\right. & \left.x_{2}, x_{3}, x_{4}\right) \\
= & \left(x_{1}, x_{2}, x_{3}\right)+\left(R_{2}\left(x_{1}, R_{1}\left(x_{2}, x_{3}\right)\right), R_{2}\left(x_{2}, x_{3}\right), x_{4}\right) \\
& +\left(x_{1}, R_{1}\left(x_{2}, x_{3}\right), R_{1}\left(R_{2}\left(x_{2}, x_{3}\right), x_{4}\right)\right)+\left(x_{2}, x_{3}, x_{4}\right) \\
& -\left\{\left(R_{1}\left(x_{1}, x_{2}\right), R_{1}\left(R_{2}\left(x_{1}, x_{2}\right), x_{3}\right), R_{1}\left(R_{2}\left(R_{2}\left(x_{1}, x_{2}\right), x_{3}\right), x_{4}\right)\right)\right. \\
& +\left(R_{2}\left(x_{1}, x_{2}\right), x_{3}, x_{4}\right)+\left(x_{1}, x_{2}, R_{1}\left(x_{3}, x_{4}\right)\right) \\
& \left.+\left(R_{2}\left(x_{1}, R_{1}\left(x_{2}, R_{1}\left(x_{3}, x_{4}\right)\right)\right), R_{2}\left(x_{2}, R_{1}\left(x_{3}, x_{4}\right)\right), R_{2}\left(x_{3}, x_{4}\right)\right)\right\} .
\end{aligned}
$$

These Fig. 4 are dual figures of movie version of the "tetrahedral move," one of the Roseman moves (analogs of Reidemeister moves for knotted surfaces, see [9]). Using the convention depicted in Fig. 2 on relation between squares and crossings, we obtain the left figure of Fig. 5 from the top figure of Fig. 4. The last figure, at the bottom in Fig. 4, corresponds to the right figure in Fig. 5. Two changes in the left and right of Fig. 4 correspond to two sequences of type III Reidemeister moves changing the left figure of Fig. 5 to the right (see [9] again). In terms of projections into 3-space of a knotted surface in 4-space, each type III move corresponds to a triple point in projection.

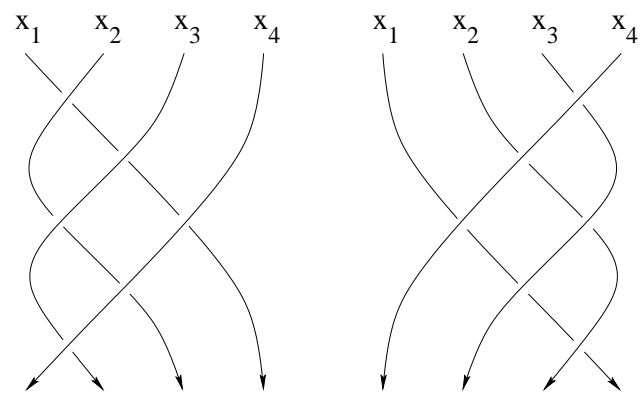

Fig. 5. Dual knot diagrams

2.3. Obstruction and extension cocycles. Low dimensional Yang-Baxter cocycles have interpretations as obstruction cocycles in extensions of YangBaxter sets. This is a situation similar to group and other cohomology theories, and generalizes the quandle cohomology case. 
Let $0 \rightarrow N \stackrel{i}{\rightarrow} G \stackrel{p}{\rightarrow} A \rightarrow 0$ be an exact sequence of abelian groups with a set-theoretic section $s: A \rightarrow G$ which is normalized in the sense that $s(0)=0$.

Let $f \in Z_{\mathrm{YB}}^{n}(X ; A)$ for a positive integer $n$. Then $\delta f\left(x_{1}, \ldots, x_{n}\right)$ has $2 n$ terms, each of which has $n-1$ arguments. Let $\delta f\left(x_{1}, \ldots, x_{n}\right)=T_{1}+$ $\cdots+T_{2 n}$ be such terms. Consider the expression $H=s\left(T_{1}\right)+\cdots+s\left(T_{2 n}\right)$. Then $p(H)=0 \in A$ since $p \circ s=\mathrm{id}, p$ is a homomorphism, and $f$ is a cocycle. Therefore there is a unique element $\psi\left(x_{1}, \ldots, x_{n}\right) \in N$ such that $i \psi\left(x_{1}, \ldots, x_{n}\right)=H$. For example, for $n=2$, we obtain

$$
s f(x)+s f(y)=i \psi(x, y)+s f\left(R_{1}(x, y)\right)+s f\left(R_{2}(x, y)\right) .
$$

Proposition 2.8. $\psi \in Z_{\mathrm{YB}}^{n+1}(X ; N)$.

Proof. The $2 n$ terms $T_{1}, \ldots, T_{2 n}$ are in one-to-one correspondence with $(n-1)$-faces of $\mathcal{I}_{n}$ whose initial path is labeled by $x_{1}, \ldots, x_{n}$ via $L$. Assign $i \psi\left(x_{1}, \ldots, x_{n}\right)$ to this cube $\mathcal{I}_{n}$. Then in $\mathcal{I}_{n+1}$ whose initial path is labeled by $\left(x_{1}, \ldots, x_{n+1}\right)$, the $n$-faces $\mathcal{I}$ are in one-to-one correspondence with the assigned $i \psi$. Then $\delta \psi=0$ follows from $\delta^{2}=0$.

We call such a cocycle $\psi$ an obstruction $(n+1)$-cocycle.

Explicit calculations can be carried out using this correspondence. For example, for $n=2$, on the one hand,

$$
\begin{aligned}
s f(x)+ & s f(y)+s f(z) \\
= & i \psi(x, y)+s f\left(R_{1}(x, y)\right)+\underline{s f\left(R_{2}(x, y)\right)+s f(z)} \\
= & i \psi(x, y)+i \psi\left(R_{2}(x, y), z\right)+\underline{s f\left(R_{1}(x, y)\right)+s f\left(R_{1}\left(R_{2}(x, y), z\right)\right)} \\
& +s f\left(R_{2}\left(R_{2}(x, y), z\right)\right) \\
= & i \psi(x, y)+i \psi\left(R_{2}(x, y), z\right)+i \psi\left(R_{1}(x, y), R_{1}\left(R_{2}(x, y), z\right)\right) \\
& +s f\left(R_{1}\left(R_{1}(x, y), R_{1}\left(R_{2}(x, y), z\right)\right)\right) \\
& +s f\left(R_{2}\left(R_{1}(x, y), R_{1}\left(R_{2}(x, y), z\right)\right)\right)+s f\left(R_{2}\left(R_{2}(x, y), z\right)\right)
\end{aligned}
$$

and on the other hand,

$$
\begin{aligned}
s f(x)+\frac{s f(y)+s f(z)}{=} & i \psi(y, z)+\underline{s f(x)+s f\left(R_{1}(y, z)\right)}+s f\left(R_{2}(y, z)\right) \\
= & i \psi(y, z)+i \psi\left(x, R_{1}(y, z)\right)+s f\left(R_{1}\left(x, R_{1}(y, z)\right)\right) \\
& \quad+\frac{s f\left(R_{2}\left(x, R_{1}(y, z)\right)\right)+s f\left(R_{2}(y, z)\right)}{=} \\
& \quad+s f(y, z)+i \psi\left(x, R_{1}(y, z)\right)+i \psi\left(R_{1}\left(x, R_{1}(y, z)\right), R_{2}(y, z)\right) \\
& \left.+s f\left(R_{2}\left(R_{2}\left(x, R_{1}(y, z)\right)\right), R_{2}(y, z)\right)\right),
\end{aligned}
$$


where the defining relation of $\psi$ was applied to underlined terms. We recover the formula for the second coboundary homomorphism this way. This computation is directly visualized from Fig. 3.

Next we consider extensions.

Proposition 2.9. Let $V=A \times X$ and $S: V \times V \rightarrow V \times V$ be defined by

$$
\begin{aligned}
& S\left(\left(a_{1}, x_{1}\right),\left(a_{2}, x_{2}\right)\right) \\
& \quad=\left(\left(a_{2}+\psi_{1}\left(x_{1}, x_{2}\right), R_{1}\left(x_{1}, x_{2}\right)\right),\left(a_{1}+\psi_{2}\left(x_{1}, x_{2}\right), R_{2}\left(x_{1}, x_{2}\right)\right)\right)
\end{aligned}
$$

for any $\left(a_{i}, x_{i}\right) \in V$. If $(V, S)$ is a Yang-Baxter set, then $\psi_{1}+\psi_{2} \in Z_{\mathrm{YB}}^{2}(X ; A)$.

Proof. We compute

$$
\begin{aligned}
(S \times 1)(1 \times & S)(S \times 1)\left(\left(a_{1}, x_{1}\right),\left(a_{2}, x_{2}\right),\left(a_{3}, x_{3}\right)\right) \\
= & \left(\left(a_{3}+\psi_{1}\left(R_{2}\left(x_{1}, x_{2}\right), x_{3}\right)+\psi_{1}\left(R_{1}\left(x_{1}, x_{2}\right), R_{1}\left(R_{2}\left(x_{1}, x_{2}\right), x_{3}\right)\right),\right.\right. \\
& \left.R_{1}\left(R_{1}\left(x_{1}, x_{2}\right), R_{1}\left(R_{2}\left(x_{1}, x_{2}\right), x_{3}\right)\right)\right), \\
& \left(a_{2}+\psi_{1}\left(x_{1}, x_{2}\right)+\psi_{2}\left(R_{1}\left(x_{1}, x_{2}\right), R_{1}\left(R_{2}\left(x_{1}, x_{2}\right), x_{3}\right)\right),\right. \\
& \left.R_{2}\left(R_{1}\left(x_{1}, x_{2}\right), R_{1}\left(R_{2}\left(x_{1}, x_{2}\right), x_{3}\right)\right)\right), \\
& \left(a_{1}+\psi_{2}\left(x_{1}, x_{2}\right)+\psi_{2}\left(R_{2}\left(x_{1}, x_{2}\right), x_{3}\right),\right. \\
& \left.\left.R_{2}\left(R_{2}\left(x_{1}, x_{2}\right), x_{3}\right)\right)\right)
\end{aligned}
$$

and on the other hand,

$$
\begin{aligned}
&(1 \times S)(S \times 1)(1 \times S)\left(\left(a_{1}, x_{1}\right),\left(a_{2}, x_{2}\right),\left(a_{3}, x_{3}\right)\right) \\
&=(\left(a_{3}+\psi_{1}\left(x_{2}, x_{3}\right)+\psi_{1}\left(x_{1}, R_{1}\left(x_{2}, x_{3}\right)\right),\right. \\
&\left.R_{1}\left(x_{1}, R_{1}\left(x_{2}, x_{3}\right)\right)\right), \\
&\left(a_{2}+\psi_{2}\left(x_{2}, x_{3}\right)+\psi_{1}\left(R_{2}\left(x_{1}, R_{1}\left(x_{2}, x_{3}\right)\right), R_{2}\left(x_{2}, x_{3}\right)\right),\right. \\
&\left.R_{1}\left(R_{2}\left(x_{1}, R_{1}\left(x_{2}, x_{3}\right)\right), R_{2}\left(x_{2}, x_{3}\right)\right)\right), \\
&\left(a_{1}+\psi_{2}\left(x_{1}, R_{1}\left(x_{2}, x_{3}\right)\right)+\psi_{2}\left(R_{2}\left(x_{1}, R_{1}\left(x_{2}, x_{3}\right)\right), R_{2}\left(x_{2}, x_{3}\right)\right),\right. \\
&\left.R_{2}\left(R_{2}\left(x_{1}, R_{1}\left(x_{2}, x_{3}\right)\right), R_{2}\left(x_{2}, x_{3}\right)\right)\right) .
\end{aligned}
$$

Hence we obtain

$$
\begin{aligned}
& \psi_{1}\left(R_{2}\left(x_{1}, x_{2}\right), x_{3}\right)+\psi_{1}\left(R_{1}\left(x_{1}, x_{2}\right), R_{1}\left(R_{2}\left(x_{1}, x_{2}\right), x_{3}\right)\right. \\
& =\psi_{1}\left(x_{2}, x_{3}\right)+\psi_{1}\left(x_{1}, R_{1}\left(x_{2}, x_{3}\right)\right), \\
& \psi_{1}\left(x_{1}, x_{2}\right)+\psi_{2}\left(R_{1}\left(x_{1}, x_{2}\right), R_{1}\left(R_{2}\left(x_{1}, x_{2}\right), x_{3}\right)\right. \\
& =\psi_{2}\left(x_{2}, x_{3}\right)+\psi_{1}\left(R_{2}\left(x_{1}, R_{1}\left(x_{2}, x_{3}\right)\right), R_{2}\left(x_{2}, x_{3}\right)\right), \\
& \psi_{2}\left(x_{1}, x_{2}\right)+\psi_{2}\left(R_{2}\left(x_{1}, x_{2}\right), x_{3}\right) \\
& =\psi_{2}\left(x_{1}, R_{1}\left(x_{2}, x_{3}\right)\right)+\psi_{2}\left(R_{2}\left(x_{1}, R_{1}\left(x_{2}, x_{3}\right)\right), R_{2}\left(x_{2}, x_{3}\right)\right),
\end{aligned}
$$


from each factor containing $a_{3}, a_{2}, a_{1}$ respectively; by adding these equalities we obtain the 2-cocycle condition for $\psi_{1}+\psi_{2}$, and the result follows.

Corollary 2.10. Suppose $A$ is a ring in which 2 is invertible. Let $V=$ $A \times X$ and $S: V \times V \rightarrow V \times V$ be defined by

$$
\begin{aligned}
S\left(\left(a_{1}, x_{1}\right)\right. & \left.,\left(a_{2}, x_{2}\right)\right) \\
& =\left(\left(a_{2}+\psi\left(x_{1}, x_{2}\right), R_{1}\left(x_{1}, x_{2}\right)\right),\left(a_{1}+\psi\left(x_{1}, x_{2}\right), R_{2}\left(x_{1}, x_{2}\right)\right)\right)
\end{aligned}
$$

for any $\left(a_{i}, x_{i}\right) \in V$. If $(V, S)$ is a Yang-Baxter set, then $\psi \in Z_{\mathrm{YB}}^{2}(X ; A)$.

Proof. In the preceding proposition, set $\psi_{1}=\psi_{2}$; then we obtain twice the cocycle condition at the end, which yields the result as 2 is invertible.

Definition 2.11. The Yang-Baxter set $(V, S)$ defined in Proposition 2.9 is called an extension of $(X, R)$ by $\left(\psi_{1}, \psi_{2}\right)$.

EXAMPLE 2.12. Let $X=\Omega_{q}=\mathbb{Z}_{q}\left[s^{ \pm 1}, t^{ \pm 1}\right] /(1-s)(1-t)$. The same matrix $R=\left[\begin{array}{cc}1-s & s \\ t & 1-t\end{array}\right]$ used in Example 2.1 defines a Yang-Baxter set structure on $\Omega_{q}$. Let $A=X=\Omega_{q}$ and define $\psi_{i}: X \times X \rightarrow A(=X)$ by $\psi_{i}(x, y)=u_{i}(y-x)$ for some $u_{i} \in X$, for $i=1,2$.

Let $V=A \times X=\left(\Omega_{q}\right)^{2}$ and consider $S: V \times V \rightarrow V \times V$ defined as in Proposition 2.9:

$$
\begin{aligned}
& S\left(\left(a_{1}, x_{1}\right),\left(a_{2}, x_{2}\right)\right) \\
& \quad=\left(\left(a_{2}+\psi_{1}\left(x_{1}, x_{2}\right), R_{1}\left(x_{1}, x_{2}\right)\right),\left(a_{1}+\psi_{2}\left(x_{1}, x_{2}\right), R_{2}\left(x_{1}, x_{2}\right)\right)\right)
\end{aligned}
$$

for any $\left(a_{i}, x_{i}\right) \in V$. Then $S$ is represented by the matrix

$$
\left[\begin{array}{cccc}
0 & -u_{1} & 1 & u_{1} \\
0 & 1-s & 0 & s \\
1 & -u_{2} & 0 & u_{2} \\
0 & t & 0 & 1-t
\end{array}\right]
$$

also denoted by $S$. Set

$$
Y=\left[\begin{array}{cc}
1 & u_{1} \\
0 & s
\end{array}\right], \quad Z=\left[\begin{array}{cc}
1 & -u_{2} \\
0 & t
\end{array}\right] .
$$

Then $S$ can be written as $S=\left[\begin{array}{cc}E-Y & Y \\ Z & E-Z\end{array}\right]$, where $Y, Z$ invertible. If $u_{2}(1-s)=0=u_{1}(1-t)$, then $Y Z=Z Y$ and $(E-Y)(E-Z)=0$, so by Example 2.1, $(V, S)$ defines a Yang-Baxter set, and is an extension of $(X, R)$.

EXAmple 2.13. Let $(X, R)$ be the Yang-Baxter set in Example 2.1. Specifically, for a ring $k$ in which 2 is invertible, let $X=k^{2}$ and $R=$ $\left[\begin{array}{cc}E-Y & Y \\ Z & E-Z\end{array}\right]$, where $R$ is a block matrix of 2 by 2 matrices with $Y=\left[\begin{array}{ll}1 & s \\ 0 & 1\end{array}\right]$ and $Z=\left[\begin{array}{ll}1 & t \\ 0 & 1\end{array}\right]$, where $s, t \in k$. 
Let $A=X=k^{2}$ and $V=A \times X$. Let $\psi: X \times X \rightarrow A(=X)$ be defined by

$$
\psi\left(\left[\begin{array}{l}
x_{1} \\
x_{2}
\end{array}\right],\left[\begin{array}{l}
y_{1} \\
y_{2}
\end{array}\right]\right)=\left[\begin{array}{c}
w\left(y_{2}-x_{2}\right) \\
0
\end{array}\right],
$$

where $w \in k$. Define $S: V \times V \rightarrow V \times V$ as in Proposition 2.9:

$$
S((\vec{a}, \vec{x}),(\vec{b}, \vec{y}))=\left(\left(\vec{b}+\psi(\vec{x}, \vec{y}), R_{1}(\vec{x}, \vec{y})\right),\left(\vec{a}+\psi(\vec{x}, \vec{y}), R_{2}(\vec{x}, \vec{y})\right)\right)
$$

for any $(\vec{a}, \vec{x}),(\vec{b}, \vec{y}) \in V$.

We now show that $(V, S)$ is an extension of $(X, R)$ by $\psi$, and as a consequence of Corollary 2.10, we obtain $\psi \in Z_{\mathrm{YB}}^{2}(X ; A)$.

The map $S$ above, on the space $(A \times X) \times(A \times X)$, can be written as a matrix

$$
\left[\begin{array}{cccc}
O & -W & E & W \\
O & E-Y & O & Y \\
E & -W & O & W \\
O & Z & O & E-Z
\end{array}\right]
$$

where $O$ denotes the zero matrix and $W=\left[\begin{array}{ll}0 & w \\ 0 & 0\end{array}\right]$. Then the matrix of $S$ is written as $\left[\begin{array}{cc}E-Y^{\prime} & Y^{\prime} \\ Z^{\prime} & E-Z^{\prime}\end{array}\right]$, where $Y^{\prime}=\left[\begin{array}{cc}E & W \\ O & Y\end{array}\right]$ and $Z^{\prime}=\left[\begin{array}{cc}E & -W \\ O & Z\end{array}\right]$. It is seen that $\left(E-Y^{\prime}\right)\left(E-Z^{\prime}\right)=O$, and $(V, S)$ is a Yang-Baxter set.

Corollary 2.10 implies that $\psi \in Z_{\mathrm{YB}}^{2}(X ; A)$ provided 2 is invertible in $k$.

Proposition 2.14. Let $k$ be a ring in which 2 is invertible, set $X=$ $k^{2}$, and $(X, R)$ be the Yang-Baxter set defined in Examples 2.1 and 2.13, with $w \neq 0$ and $s=t$, that is, $R=\left[\begin{array}{cc}E-Y & Y \\ Y & E-Y\end{array}\right], Y=\left[\begin{array}{ll}1 & t \\ 0 & 1\end{array}\right]=Z$. Then $H_{\mathrm{YB}}^{2}(X ; A) \neq 0$.

Proof. Note that

$$
\psi\left(\left[\begin{array}{l}
1 \\
0
\end{array}\right],\left[\begin{array}{l}
0 \\
1
\end{array}\right]\right)=\left[\begin{array}{l}
w \\
0
\end{array}\right], \quad \psi\left(\left[\begin{array}{l}
0 \\
1
\end{array}\right],\left[\begin{array}{l}
1 \\
0
\end{array}\right]\right)=\left[\begin{array}{c}
-w \\
0
\end{array}\right],
$$

so that they take distinct values, since $w \neq 0$ and 2 is invertible in $k$.

On the other hand, suppose $\psi=\delta f$ for some $f \in C_{\mathrm{YB}}^{1}(X ; A)$. One computes that

$$
\begin{aligned}
(\delta f)\left(\left[\begin{array}{l}
1 \\
0
\end{array}\right],\left[\begin{array}{l}
0 \\
1
\end{array}\right]\right)= & f\left(\left[\begin{array}{l}
1 \\
0
\end{array}\right]\right)+f\left(\left[\begin{array}{l}
0 \\
1
\end{array}\right]\right) \\
& -f\left(\left[\begin{array}{l}
t \\
1
\end{array}\right]\right)-f\left(\left[\begin{array}{c}
-t+1 \\
0
\end{array}\right]\right) \\
= & (\delta f)\left(\left[\begin{array}{l}
0 \\
1
\end{array}\right],\left[\begin{array}{l}
1 \\
0
\end{array}\right]\right),
\end{aligned}
$$

which is a contradiction. 
We consider another family of examples that are similar to those considered in [4].

Proposition 2.15. For positive integers $q, h, k$, let

$$
X=\Omega_{q}^{(h, k)}=\mathbb{Z}_{q}\left[s^{ \pm 1}, t^{ \pm 1}\right] /\left\{(1-s)^{h},(1-t)^{k}\right\} .
$$

Let $R: X \times X \rightarrow X \times X$ be represented by the matrix $\left[\begin{array}{cc}1-s & s \\ t & 1-t\end{array}\right]$. Then $V=\Omega_{q}^{(h+1, k+1)}$ is an extension of $\Omega_{q}^{(h, k)}$, where $S: V \times V \rightarrow V \times V$ is represented by the same matrix that defines $R$, and $A=\mathbb{Z}_{q} \times \mathbb{Z}_{q}$.

Proof. Recall that $\Omega_{q}=\mathbb{Z}_{q}\left[t^{ \pm 1}, s^{ \pm 1}\right] /(1-s)(1-t)$. Represent elements $\alpha, \beta \in V$ by

$$
\begin{aligned}
& \alpha=\alpha_{0}+\sum_{i=1}^{h} \alpha_{i}(1-s)^{i}+\sum_{j=1}^{k} \alpha_{j}^{\prime}(1-t)^{j}, \\
& \beta=\beta_{0}+\sum_{i=1}^{h} \beta_{i}(1-s)^{i}+\sum_{j=1}^{k} \beta_{j}^{\prime}(1-t)^{j} .
\end{aligned}
$$

Let $\bar{\alpha}=p(\alpha)$ and $\bar{\beta}=p(\beta)$, where $p: V \rightarrow X$ is the natural projection. Let $\sigma: X \rightarrow V$ be the natural set-theoretic section defined by

$$
\begin{aligned}
\sigma\left(\alpha_{0}+\sum_{i=1}^{h-1} \alpha_{i}(1-s)^{i}+\sum_{j=1}^{k-1} \alpha_{j}^{\prime}(1-t)^{j}\right) & \\
& =\alpha_{0}+\sum_{i=1}^{h} \alpha_{i}(1-s)^{i}+\sum_{j=1}^{k} \alpha_{j}^{\prime}(1-t)^{j},
\end{aligned}
$$

where $\alpha_{h}=\alpha_{k}^{\prime}=0$. We identify $V$ with $A \times X$ by means of $f: V \rightarrow A \times X$, where

$$
f\left(\alpha_{0}+\sum_{i=1}^{h} \alpha_{i}(1-s)^{i}+\sum_{j=1}^{k} \alpha_{j}^{\prime}(1-t)^{j}\right)=\left(\left(\alpha_{h}, \alpha_{k}^{\prime}\right), \bar{\alpha}\right) .
$$

Then we compute that

$$
\begin{aligned}
S\left(\left(\left(\alpha_{h}, \alpha_{k}^{\prime}\right), \bar{\alpha}\right),\left(\left(\beta_{h}, \beta_{k}^{\prime}\right), \bar{\beta}\right)\right) & \\
= & S(\alpha, \beta) \\
= & ((1-s) \alpha+(1-(1-s)) \beta,(1-(1-t)) \alpha+(1-t) \beta) \\
= & (\beta+(1-s)(\alpha-\beta), \alpha+(1-t)(\beta-\alpha)) \\
= & \left(\left(\beta_{h}+\left(\alpha_{h-1}-\beta_{h-1}\right)(1-s)^{h}+\beta_{k}^{\prime}(1-t)^{k}+\sigma\left(S_{1}(\bar{\alpha}, \bar{\beta})\right),\right.\right. \\
& \quad \alpha_{h}(1-s)^{h}+\left(\alpha_{k}^{\prime}+\left(\beta_{k-1}^{\prime}-\alpha_{k-1}^{\prime}\right)(1-t)^{k}+\sigma\left(S_{2}(\bar{\alpha}, \bar{\beta})\right)\right)
\end{aligned}
$$




$$
\begin{aligned}
= & \left(\left(\beta_{h}+\left(\alpha_{h-1}-\beta_{h-1}\right), \beta_{k}^{\prime}, S_{1}(\bar{\alpha}, \bar{\beta})\right),\right. \\
& \left.\left(\alpha_{h}, \alpha_{k}^{\prime}+\left(\beta_{k-1}^{\prime}-\alpha_{k-1}^{\prime}\right), S_{2}(\bar{\alpha}, \bar{\beta})\right)\right) \\
= & \left(\left(\left(\beta_{h}, \beta_{k}^{\prime}\right)+\psi_{1}(\bar{\alpha}, \bar{\beta}), S_{1}(\bar{\alpha}, \bar{\beta})\right),\right. \\
& \left.\left(\left(\alpha_{h}, \alpha_{k}^{\prime}\right)+\psi_{2}(\bar{\alpha}, \bar{\beta}), S_{2}(\bar{\alpha}, \bar{\beta})\right)\right),
\end{aligned}
$$

where

$$
\begin{aligned}
& \psi_{1}(\bar{\alpha}, \bar{\beta})=\left(\alpha_{h-1}-\beta_{h-1}, 0\right) \in A=\mathbb{Z}_{q} \times \mathbb{Z}_{q}, \\
& \psi_{2}(\bar{\alpha}, \bar{\beta})=\left(0, \beta_{k-1}^{\prime}-\alpha_{k-1}^{\prime}\right) \in A=\mathbb{Z}_{q} \times \mathbb{Z}_{q},
\end{aligned}
$$

so that $V$ has the desired description, and explicit formulas for $\psi_{1}$ and $\psi_{2}$ are also obtained.

\section{The Yang-Baxter cocycle knot invariants}

3.1. A brief review of biquandles and virtual knots. A quandle, $X$, is a set with a binary operation $(a, b) \mapsto a * b$ such that

(I) For any $a \in X, a * a=a$.

(II) For any $a, b \in X$, there is a unique $c \in X$ such that $a=c * b$.

(III) For any $a, b, c \in X$, we have $(a * b) * c=(a * c) *(b * c)$.

A rack is a set with a binary operation that satisfies (II) and (III). Racks and quandles have been studied in, for example, [2, 14, 21, 23, 30]. Generalizations of racks and quandles have been studied in several papers. Here we follow descriptions in $[1,13]$.

Definition $3.1([1,13])$. A set $X$ is called a birack if there is a mapping $R: X \times X \rightarrow X \times X$ with the following properties.

1. The map $R$ is invertible. The inverse of $R$ is denoted by $\bar{R}: X \times X \rightarrow$ $X \times X$. The images of the map $R$ are written as

$$
R\left(A_{1}, A_{2}\right)=\left(R_{1}\left(A_{1}, A_{2}\right), R_{2}\left(A_{1}, A_{2}\right)\right)=\left(A_{3}, A_{4}\right),
$$

where $A_{i} \in X$ for $i=1,2,3,4$.

2. For any $A_{1}, A_{3} \in X$ there is a unique $A_{2} \in X$ such that $R_{1}\left(A_{1}, A_{2}\right)$ $=A_{3}$. We say that $R_{1}$ is left-invertible.

3. For any $A_{2}, A_{4} \in X$ there is a unique $A_{1} \in X$ such that $R_{2}\left(A_{1}, A_{2}\right)$ $=A_{4}$. We say that $R_{2}$ is right-invertible.

4. $R$ satisfies the set-theoretic Yang-Baxter equation:

$$
(R \times 1)(1 \times R)(R \times 1)=(1 \times R)(R \times 1)(1 \times R),
$$

where 1 denotes the identity mapping.

To specify the map, we also say that $(X, R)$ is a birack. 

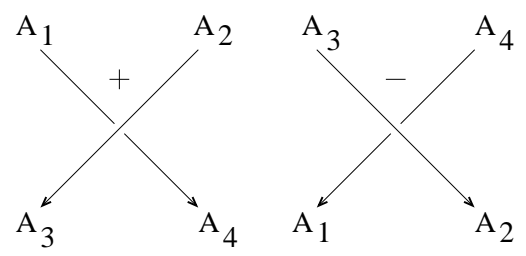

Fig. 6. A coloring by birack elements

Definition $3.2([1,13])$. A biquandle $(X, R)$ is a birack with the following property, called the type I condition.

Given an element $a$ in $X$, there exists a unique $x \in X$ such that $x=$ $R_{1}(x, a)$ and $a=R_{2}(x, a)$.

REMARK 3.3. The definition of the type I condition in $[1,13]$ is differently formulated but they prove that their definition is equivalent to the above condition. They also prove that the condition is equivalent to that, given $a$ in $X$, there exists a unique $y \in X$ such that $y=R_{2}(a, y)$ and $a=R_{1}(a, y)$. In particular, one of these implies both.

EXAmple 3.4. Those Yang-Baxter sets described in Example 2.1 define biquandles.

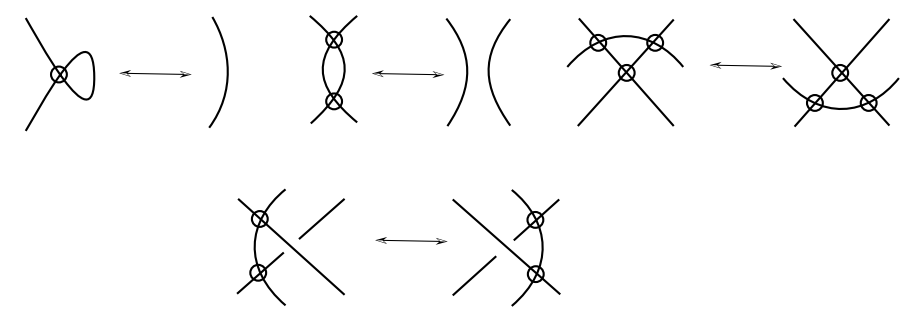

Fig. 7. Virtual Reidemeister moves

A virtual link diagram $[24,25]$ consists of generic closed curves in $\mathbb{R}^{2}$ such that each crossing is either a classical crossing with over- and under-arcs, or a virtual crossing without over or under information. Let $\mathcal{V} \mathcal{L}$ be the set of virtual link diagrams. Virtual Reidemeister equivalence is an equivalence relation on $\mathcal{V} \mathcal{L}$ generated by the Reidemeister moves depicted in Fig. 7, and ordinary Reidemeister moves. Put $V L=\mathcal{V} \mathcal{L} / \sim$, where $\sim$ is the virtual Reidemeister equivalence. Each element of $V L$ is called a virtual link.

If the given set of curves of a diagram is connected (i.e., the diagram consists of a single component curve), then it is called a virtual knot diagram. The set of virtual knot diagrams is denoted by $\mathcal{V K}$, and the set of equivalence classes is denoted by $V K=\mathcal{V K} / \sim$; its elements are called virtual knots.

Let $K$ be an oriented virtual link diagram. A diagram is immersed circles with crossing information (over and under arcs) specified at each crossing. 
The under-arcs are broken into two pieces at a crossing to specify the crossing information. In this case, the rest consists of arcs, called over-arcs of the diagram. The family of underlying immersed circles is called a projection. When crossing points are deleted from the projection, we obtain a set of open arcs, denoted by $\mathcal{A}$. In this case, over-arcs are also broken at a crossing.

Definition 3.5. Let $(X, R)$ be a birack. Let $K$ be an oriented link diagram, and $\mathcal{A}$ the set of its open arcs. A map $\mathcal{C}: \mathcal{A} \rightarrow X$ is called a coloring of $K$ by $X$ if it satisfies the following conditions.

Let $A_{i}, i=1,2,3,4$, be the images of open arcs near a positive crossing as depicted in the left of Fig. 6 under the map $\mathcal{C}$, so that they are elements of $X$ (regarded as being assigned to each arc). Then it is required that $R\left(A_{1}, A_{2}\right)=\left(A_{3}, A_{4}\right)$. At a negative crossing, the elements $A_{i}$ as depicted in the right of Fig. 6 are required to satisfy $\bar{R}\left(A_{1}, A_{2}\right)=\left(A_{3}, A_{4}\right)$. At a virtual crossing the colorings do not change for either arcs involved.

The images $A_{i}$ are called colors.

It is proved in [13] that the number of colorings of a virtual knot diagram by a biquandle does not depend on the choice of a diagram, so that the number of colorings is an invariant of virtual knots. In fact, it was shown that there is a one-to-one correspondence between the set of colorings before and after each Reidemeister or virtual Reidemeister move, such that the colors outside of a small neighborhood where the move occurs are fixed before and after the move.

3.2. Yang-Baxter cocycle invariants. In this section we define knot invariants by state-sum, using Yang-Baxter cocycles. They generalize the quandle cocycle invariant [5], and include it as a special case. The latter has been generalized to knotted surfaces in 4-space, and used for topological applications $[5,32]$, so developments into such directions are expected for the Yang-Baxter cocycle invariants as well.

In the case of quandle cocycle invariants, we needed to define "quandle condition" [5] for rack cocycles so that the state-sum is invariant under type I Reidemeister move. We need the following condition for Yang-Baxter cocycles for this purpose.

Definition 3.6. Let $(X, A)$ be a biquandle, and $A$ be an abelian group. Recall from Definition 3.2 and Remark 3.3 that for any $a \in X$ there are unique $x, y \in X$ such that $x=R_{1}(x, a), a=R_{2}(x, a), y=R_{2}(a, y)$, and $a=R_{1}(a, y)$. A Yang-Baxter 2-cocycle $\psi \in Z_{\mathrm{YB}}^{2}(X ; A)$ is said to satisfy the type $I$ condition if $\psi(x, a)=0$ and $\psi(a, y)=0$ for any $a \in X$.

Let $K$ be a classical knot or link diagram. Let a finite biquandle $(X, R)$ and a 2-cocycle $\psi \in Z_{\mathrm{YB}}^{2}(X ; A)$ be given, where $A$ is an abelian group. Let $\mathcal{C}$ denote a coloring $\mathcal{C}: \mathcal{E} \rightarrow X$, where $\mathcal{E}$ denotes the set of open arcs of $K$. 

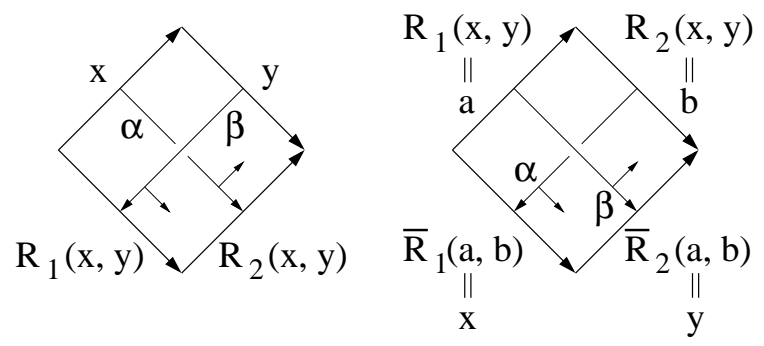

Fig. 8. Crossings of classical knot diagrams

Fix normals to the arcs in such a way that the (tangent, normal) matches the orientation of the plane (see Fig. 8). Note that the edges of the squares dual to the crossings have the parallel orientations to the normals, while tangents may not.

Let $\alpha$ be the under-arc away from which the normal to the over-arc points. Let $\beta$ be the over-arc towards which the normal to the under-arc points. Let $\mathcal{C}(\alpha)=x$ and $\mathcal{C}(\beta)=y$ (see Fig. 8). A (Boltzmann) weight, $B(\tau, \mathcal{C})$ (that depends on $\psi$ ), at a crossing $\tau$ is defined by $B(\tau, \mathcal{C})=\psi(x, y)^{\varepsilon(\tau)}$, where $\varepsilon(\tau)=1$ or -1 if $\tau$ is positive or negative, respectively.

The (Yang-Baxter) cocycle knot invariant is defined by the state-sum expression

$$
\Phi_{\mathrm{YB}}(K)=\sum_{\mathcal{C}} \prod_{\tau} B(\tau, \mathcal{C}) .
$$

The product is taken over all crossings of the given diagram $K$, and the sum is taken over all possible colorings. The values of the state-sum are taken to be in the group ring $\mathbb{Z}[A]$, where $A$ is the coefficient group written multiplicatively. The state-sum depends on the choice of the 2-cocycle $\psi$.

By checking all the Reidemeister moves as in [5], we obtain

Proposition 3.7. If $\psi \in Z_{\mathrm{YB}}^{2}(X ; A)$ satisfies the type I condition, then $\Phi_{\mathrm{YB}}(K)$ does not depend on the choice of a diagram. Hence $\Phi_{\mathrm{YB}}(K)$ defines a knot invariant.

Proposition 3.8. Let $\psi \in Z_{\mathrm{YB}}^{2}(X ; N)$ be an obstruction cocycle as in (1). Then the value of the Yang-Baxter cocycle knot invariant $\Phi_{\mathrm{YB}}(K)$ with $\psi$ is a positive integer for any classical or virtual knot or a link $K$.

Proof. The proof is similar to the one found in [5]. Choose and fix any coloring of a given knot diagram by a biquandle. Suppose $f \in Z_{\mathrm{YB}}^{2}(X ; A)$ is as in (1). Since $i: N \rightarrow G$ is injective, compute the state-sum in $\mathbb{Z}[G]$ which contains $\mathbb{Z}[N]$. Then the weight assigned at a crossing, say, $\tau$ is $i \psi(x, y)^{\varepsilon}$, where $\varepsilon=1$ or -1 depending on whether $\tau$ is positive or negative, respectively. Assign the terms $s f(x)^{\varepsilon}, s f(y)^{\varepsilon}, s f\left(R_{1}(x, y)\right)^{-\varepsilon}$, and $s f\left(R_{2}(x, y)\right)^{-\varepsilon}$ on the strings near the crossing $\tau$ colored with $x, y, R_{1}(x, y)$, and $R_{2}(x, y)$, 
respectively. Then the state-sum is computed by using these weights. However, the weights assigned to the two ends of each arc cancel, giving 1 as the contribution to the state-sum from this coloring.

3.3. Computations. In [1], it was pointed out that if a matrix $R=\left[\begin{array}{ll}A & B \\ C & D\end{array}\right]$ satisfies the YBE, then so does $R=\left[\begin{array}{cc}A & u B \\ u^{-1} C & D\end{array}\right]$ for any invertible element $u$ in the center of the ring used. In particular, consider such variants $R=$ $\left[\begin{array}{cc}1-s & u s \\ u^{-1} t & 1-t\end{array}\right]$ of those in Example 2.1. This matrix defines a biquandle structure on $\mathbb{Z}_{q}$ for a positive integer $q$ if $(1-s)(1-t) \equiv 0(\bmod q)$ and $s, t, u$ are all invertible. In this section we present some calculations for this type of biquandles.

EXAMPLE 3.9. We computed the number of colorings of Kishino-type knots (see, for example, $[1,13,28]$ for $q=15, s=4, t=11$, and $u=$ $2,4,7,8,11,13,14$. Kishino's knot is a connected sum of diagrams of the unknot, and we took three variants usually considered $\left(K_{1}\right.$ through $\left.K_{3}\right)$ and further variants $\left(K_{4}\right.$ through $\left.K_{6}\right)$, represented by the closed braid form of the following braid words. We use the usual notation $\sigma_{i}$ for a standard braid generator between $i$ th and $(i+1)$ st strings, and the notation $v_{i}$ for virtual crossing between them. The results are summarized in the table below. We included these examples for noting that the numbers of colorings alone distinguish some of these from others, as well as from the unknot.

$$
\begin{aligned}
& K_{1}=\sigma_{1} v_{1} \sigma_{1}^{-1} \sigma_{2} \sigma_{1} v_{1} \sigma_{1}^{-1} \sigma_{2}^{-1}, \\
& K_{2}=\sigma_{1} v_{1} \sigma_{1}^{-1} \sigma_{2} \sigma_{1}^{-1} v_{1} \sigma_{1} \sigma_{2}^{-1}, \\
& K_{3}=\sigma_{1}^{-1} v_{1} \sigma_{1} \sigma_{2} \sigma_{1}^{-1} v_{1} \sigma_{1} \sigma_{2}^{-1}, \\
& K_{4}=\sigma_{1} \sigma_{1} v_{1} \sigma_{1}^{-1} \sigma_{1}^{-1} \sigma_{2} \sigma_{1} \sigma_{1} v_{1} \sigma_{1}^{-1} \sigma_{1}^{-1} \sigma_{2}^{-1}, \\
& K_{5}=\sigma_{1} \sigma_{1} v_{1} \sigma_{1}^{-1} \sigma_{1}^{-1} \sigma_{2} \sigma_{1}^{-1} \sigma_{1}^{-1} v_{1} \sigma_{1} \sigma_{1} \sigma_{2}^{-1}, \\
& K_{6}=\sigma_{1}^{-1} \sigma_{1}^{-1} v_{1} \sigma_{1} \sigma_{1} \sigma_{2} \sigma_{1}^{-1} \sigma_{1}^{-1} v_{1} \sigma_{1} \sigma_{1} \sigma_{2}^{-1},
\end{aligned}
$$

Table 1. A table of numbers of colorings of Kishino-type knots

\begin{tabular}{rrrrrrr}
\hline$u$ & $K_{1}$ & $K_{2}$ & $K_{3}$ & $K_{4}$ & $K_{5}$ & $K_{6}$ \\
\hline 2 & 225 & 15 & 75 & 15 & 15 & 45 \\
4 & 15 & 15 & 45 & 45 & 15 & 75 \\
7 & 75 & 15 & 225 & 45 & 15 & 15 \\
8 & 45 & 15 & 15 & 75 & 15 & 225 \\
11 & 45 & 15 & 15 & 75 & 15 & 45 \\
13 & 15 & 15 & 45 & 225 & 15 & 75 \\
14 & 45 & 15 & 15 & 15 & 15 & 225 \\
\hline
\end{tabular}


EXAMPLE 3.10. Let $X$ be the biquandle determined by the matrix $R=$ $\left[\begin{array}{ll}0 & 2 \\ 1 & 2\end{array}\right]$ in $\mathbb{Z}_{3}$ as in the preceding example, where $q=3, s=1, t=2$, and $u=2$. Then we obtain a non-trivial 2-cocycle $f \in Z_{\mathrm{YB}}^{2}\left(X ; \mathbb{Z}_{3}\right)$ with the following values:

$$
\begin{aligned}
& f(1,0)=q_{1}, \quad f(2,2)=q_{2}, \quad f(1,1)=q_{3}, \quad f(2,0)=-q_{1}, \\
& f(0,2)=q_{1}-q_{3}, \quad f(0,1)=-q_{1}-q_{2},
\end{aligned}
$$

where $q_{i}, i=1,2,3$, are arbitrary choices of numbers modulo 3 . The values for unspecified evaluations are zeros. This cocycle was obtained by Maple and confirmed independently by Mathematica. This small biquandle of three elements already takes non-trivial values for the cocycle invariant on virtual knots. We obtain, for example, the following by direct calculations:

$$
\Phi_{\mathrm{YB}}\left(\operatorname{cl}\left(\sigma_{1}^{n} v_{1}\right)\right)= \begin{cases}3 & \text { if } n \equiv 0(\bmod 3), \\ 1+\xi^{q_{1}}+\xi^{-q_{1}} & \text { if } n \equiv 1,2(\bmod 3) .\end{cases}
$$

Here, $\xi$ denotes a multiplicative generator of the coefficient group $\mathbb{Z}_{3}$.

EXAMPLE 3.11. Let $X$ be the biquandle determined by the matrix $R=$ $\left[\begin{array}{cc}0 & -1 \\ 1 & 2\end{array}\right]$ in $\mathbb{Z}_{4}$ as before, where $q=4, s=1, t=-1$, and $u=-1$. Then we obtain a non-trivial 2-cocycle $f \in Z_{\mathrm{YB}}^{2}\left(X ; \mathbb{Z}_{4}\right)$ with the following values:

$$
\begin{aligned}
& f(0,1)=f(1,1)=f(1,2)=f(3,3)=1, \quad f(0,2)=2, \\
& f(1,0)=f(2,1)=f(3,0)=f(3,2)=3 .
\end{aligned}
$$

The values for unspecified evaluations are zeros. This cocycle was again obtained by Maple and confirmed independently by Mathematica.

Let $\xi$ denote a generator of the coefficient group $\mathbb{Z}_{4}$. For the torus links (the closure $\operatorname{cl}\left(\sigma_{1}^{n}\right)$ of 2-braids, $n \in \mathbb{Z}$ ), we obtain the following formula:

$$
\Phi_{\mathrm{YB}}\left(\operatorname{cl}\left(\sigma_{1}^{n}\right)\right)= \begin{cases}4 & \text { if } n \equiv 1(\bmod 2), \\ 4+4 \xi^{2} & \text { if } n \equiv 2(\bmod 4), \\ 8+8 \xi^{3} & \text { if } n \equiv 4(\bmod 16), \\ 8+8 \xi^{2} & \text { if } n \equiv 8(\bmod 16), \\ 8+8 \xi & \text { if } n \equiv 12(\bmod 16), \\ 16 & \text { if } n \equiv 0(\bmod 16) .\end{cases}
$$

Proof. Since $R^{4}$ is the identity matrix, and the coefficient group is $\mathbb{Z}_{4}$, it is clear that the cocycle invariant has period 16: $\Phi_{\mathrm{YB}}\left(\operatorname{cl}\left(\sigma_{1}^{n+16}\right)\right)=\Phi_{\mathrm{YB}}\left(\operatorname{cl}\left(\sigma_{1}^{n}\right)\right)$ for any $n \in \mathbb{Z}$. When $n \equiv 1(\bmod 2)$, the closed braid $\operatorname{cl}\left(\sigma_{1}^{n}\right)$ is colored if and only if the colors at the top strings are of the form $(a,-a), a \in X$. This can be seen from the matrix $R^{n}$. For each such coloring the contribution at each crossing is zero, so that $\Phi_{\mathrm{YB}}\left(\operatorname{cl}\left(\sigma_{1}^{n}\right)\right)=4$. Now if $n \equiv 2(\bmod 4)$ then the closed braid is colored if and only if the colors at the top arcs are of the form $(a,-a)$ or $(a, 2-a), a \in X$. Any coloring of the form $(a, 2-a)$ 
gives a total contribution of two. This implies that $\Phi_{\mathrm{YB}}\left(\operatorname{cl}\left(\sigma_{1}^{n}\right)\right)=4+4 \xi^{2}$. There are 16 possible colorings if $n \equiv 4(\bmod 16)$, for all pairs $(a, b)$ of top arcs. For the colors of the form $(a,-a)$, the contribution is zero. For $(a, 2-a)$, the contribution is doubled compared to the case $n \equiv 2(\bmod 4)$, so that the contribution is also zero. The remaining colors contribute 3 , so that $\Phi_{\mathrm{YB}}\left(\operatorname{cl}\left(\sigma_{1}^{n}\right)\right)=8+8 \xi^{3}$. The contributions of these remaining colors get doubled, tripled, and quadrupled, respectively, for the cases $n \equiv 8,12$, and $0 \bmod 16$.

In particular, $\Phi_{\mathrm{YB}}\left(\operatorname{cl}\left(\sigma_{1}^{4}\right)\right)=8+8 \xi^{3}$ and $\Phi_{\mathrm{YB}}\left(\operatorname{cl}\left(\sigma_{1}^{-4}\right)\right)=8+8 \xi$ so that the invariant detects the chirality.

The invariant can take other values for some virtual knots, such as:

$$
\Phi_{\mathrm{YB}}\left(\operatorname{cl}\left(\sigma_{1} v_{1}\right)^{n}\right)= \begin{cases}3+\xi+\xi^{2}+3 \xi^{3} & \text { if } n \equiv 1(\bmod 4), \\ 6+6 \xi^{2}+2 \xi+2 \xi^{3} & \text { if } n \equiv 2(\bmod 4), \\ 3+3 \xi+\xi^{2}+\xi^{3} & \text { if } n \equiv 3(\bmod 4), \\ 12+4 \xi^{2} & \text { if } n \equiv 4(\bmod 8), \\ 16 & \text { if } n \equiv 0(\bmod 8) .\end{cases}
$$

The invariant can be non-trivial for links with zero linking number. The Borromean rings, a three-component link with pairwise linking number zero and represented as the closed braid of $\left(\sigma_{1}^{-1} \sigma_{2}\right)^{3}$, has the value $16+48 \xi^{2}$.

These calculations suggest that these invariants are quite non-trivial and deserve further study.

\section{References}

[1] A. Bartholomew and R. Fenn, Quaternionic invariants of virtual knots and links, preprint found at http://www.maths.sussex.ac.uk///Staff/RAF/Maths/.

[2] E. Brieskorn, Automorphic sets and singularities, in: Contemp. Math. 78, Amer. Math. Soc., 1988, 45-115.

[3] S. Budden and R. Fenn, The equation $[B,(A-1)(A, B)]=0$ and virtual knots and links, this volume, 19-29.

[4] J. S. Carter, M. Elhamdadi, M. A. Nikiforou and M. Saito, Extensions of quandles and cocycle knot invariants, J. Knot Theory Ramifications 12 (2003), 725-738.

[5] J. S. Carter, D. Jelsovsky, S. Kamada, L. Langford and M. Saito, Quandle cohomology and state-sum invariants of knotted curves and surfaces, Trans. Amer. Math. Soc. 355 (2003), 3947-3989.

[6] J. S. Carter, D. Jelsovsky, S. Kamada and M. Saito, Computations of quandle cocycle invariants of knotted curves and surfaces, Adv. Math. 157 (2001), 36-94.

[7] - , - - - , Quandle homology groups, their Betti numbers, and virtual knots, J. Pure Appl. Algebra 157 (2001), 135-155.

[8] J. S. Carter, S. Kamada and M. Saito, Geometric interpretations of quandle homology, J. Knot Theory Ramifications 10 (2001), 345-386.

[9] J. S. Carter and M. Saito, Knotted Surfaces and Their Diagrams, Amer. Math. Soc., 1998. 
[10] J. S. Carter and M. Saito, Diagrammatic invariants of knotted curves and surfaces, unpublished note written in 1992 with limited circulation, on which this paper is based.

[11] P. Etingof, T. Schedler and A. Soloviev, Set-theoretical solutions of the quantum Yang-Baxter equation, Duke Math. J. 100 (1999), 169-209.

[12] P. Etingof, A. Soloviev and R. Guralnick, Indecomposable set-theoretical solutions to the quantum Yang-Baxter equation on a set with a prime number of elements, J. Algebra 242 (2001), 709-719.

[13] R. Fenn, M. Jordan-Santana and L. H. Kauffman, Biquandles and virtual links, Topology Appl. 145 (2004), 157-175.

[14] R. Fenn and C. Rourke, Racks and links in codimension two, J. Knot Theory Ramifications 1 (1992), 343-406.

[15] R. Fenn, C. Rourke and B. Sanderson, An introduction to species and the rack space, in: Topics in Knot Theory (Erzurum, 1992), NATO Adv. Sci. Inst. Ser. C Math. Phys. Sci. 399, Kluwer, Dordrecht, 1993, 33-55.

[16] —, - - , Trunks and classifying spaces, Appl. Categ. Structures 3 (1995), 321-356.

[17] —, - - - James bundles, Proc. London Math. Soc. (3) 89 (2004), 217-240.

[18] R. H. Fox, A quick trip through knot theory, in: Topology of 3-Manifolds, M. K. Fort Jr. (ed.), Prentice-Hall, 1962, 120-167.

[19] M. Goussarov, M. Polyak and O. Viro, Finite-type invariants of classical and virtual knots, Topology 39 (2000), 1045-1068.

[20] V. F. R. Jones, Hecke algebra representations of braid groups and link polynomials, Ann. of Math. 126 (1989), 335-388.

[21] D. Joyce, A classifying invariant of knots, the knot quandle, J. Pure Appl. Algebra 23 (1982), 37-65.

[22] N. Kamada and S. Kamada, Abstract link diagrams and virtual knots, J. Knot Theory Ramifications 9 (2000), 93-106.

[23] L. H. Kauffman, Knots and Physics, Ser. Knots Everything 1, World Sci., 1991.

[24] —, Virtual knots, talks at MSRI Meeting in January 1997 and AMS Meeting at University of Maryland, College Park in March 1997.

[25] —, Virtual knot theory, European J. Combin. 20 (1999), 663-690.

[26] L. H. Kauffman and D. E. Radford, Bi-oriented quantum algebras, and a generalized Alexander polynomial for virtual links, in: Diagrammatic Morphisms and Applications (San Francisco, CA, 2000), Contemp. Math., 318, Amer. Math. Soc., Providence, RI, 2003, 113-140.

[27] S.-G. Kim, Virtual knot groups and their peripheral structure, J. Knot Theory Ramifications 9 (2000), 797-812.

[28] T. Kishino and S. Satoh, A note on non-classical virtual knots, ibid. 13 (2004), 845-856.

[29] J.-H. Lu, M. Yan and Y.-C. Zhu, On the set-theoretical Yang-Baxter equation, Duke Math. J. 104 (2000), 1-18.

[30] S. Matveev, Distributive groupoids in knot theory, Mat. Sb. (N.S.) 119 (1982), 78-88, 160 (in Russian).

[31] C. Rourke and B. Sanderson, There are two 2-twist-spun trefoils, preprint at http://xxx.lanl.gov/abs/math.GT/0006062.

[32] S. Satoh and A. Shima, The 2-twist spun trefoil has the triple point number four, Trans. Amer. Math. Soc. 356 (2004), 1007-1024.

[33] J. Sawollek, On Alexander-Conway polynomials for virtual knots and links, preprint, arXiv:math.GT/9912173. 
[34] D. Silver and S. Williams, Alexander groups and virtual links, J. Knot Theory Ramifications 10 (2001), 151-160.

[35] - - - A generalized Burau representation for string links, Pacific J. Math. 197 (2001), 241-255.

[36] A. Soloviev, Non-unitary set-theoretical solutions to the quantum Yang-Baxter equation, Math. Res. Lett. 7 (2000), 577-596.

[37] V. Turaev, The Yang-Baxter equation and invariants of links, Invent. Math. 92 (1988), 527-553.

[38] D. Rolfsen, Knots and Links, Publish or Perish, Berkeley, 1976.

[39] D. Roseman, Reidemeister-type moves for surfaces in four dimensional space, in: Knot Theory, Banach Center Publ. 42, Inst. Math., Polish Acad. Sci., 1998, 347-380.

[40] M. Wada, Group invariants of links, Topology 31 (1992), 399-406.

University of South Alabama

Mobile, AL 36688, U.S.A.

E-mail: carter@jaguar1.usouthal.edu
University of South Florida

Tampa, FL 33620, U.S.A.

E-mail: emohamed@math.usf.edu saito@math.usf.edu

Received 7 January 2004;

in revised form 2 December 2004 